\title{
A new swarm intelligence information technique for improving information balancedness on the skin lesions segmentation
}

\author{
H. J. Abd ${ }^{1}$, Ahmad S. Abdullah ${ }^{2}$, Muhammed Salah Sadiq Alkafaji ${ }^{3}$ \\ ${ }^{1}$ Department of Electrical Engineering, College of Engineering, Babylon University, Iraq \\ ${ }^{2}$ Department of Communications, College of Engineering, University of Diyala, Iraq \\ ${ }^{3}$ Department of Electrical Power Techniques Engineering, Technical College/Al- Mausaib, \\ Al-Furat Al-Awsat Technical University, Iraq
}

\begin{tabular}{l}
\hline \hline Article Info \\
\hline Article history: \\
Received Aug 20, 2019 \\
Revised May 8, 2020 \\
Accepted May 23, 2020 \\
\hline
\end{tabular}

Keywords:

Automatic segmentation

Dermatologists

Median filter

Skin lesion

Swarm intelligence techniques

\begin{abstract}
Methods of image processing can recognize the images of melanoma lesions border in addition to the disease compared to a skilled dermatologist. New swarm intelligence technique depends on meta-heuristic that is industrialized to resolve composite real problems which are problematic to explain by the available deterministic approaches. For an accurate detection of all segmentation and classification of skin lesions, some dealings should be measured which contain, contrast broadening, irregularity quantity, choice of most optimal features, and so into the world. The price essential for the action of progressive disease cases is identical high and the survival percentage is low. Many electronic dermoscopy classifications are advanced depend on the grouping of form, surface and dye features to facilitate premature analysis of malignance. To overcome this problematic, an effective prototypical for accurate boundary detection and arrangement is obtainable. The projected classical recovers the optimization segment of accuracy in its pre-processing stage, applying contrast improvement of lesion area compared to the contextual. In conclusion, optimized features are future fed into of artifical bee colony $(\mathrm{ABC})$ segmentation. Wide-ranging researches have been supported out on four databases named as, ISBI $(2016,2017,2018)$ and PH2. Also, the selection technique outclasses and successfully indifferent the dismissed features. The paper shows a different process for lesions optimal segmentation that could be functional to a variation of images with changed possessions and insufficiencies is planned with multistep pre-processing stage.
\end{abstract}

Copyright $\odot 2020$ Institute of Advanced Engineering and Science. All rights reserved.

Corresponding Author:

H. J. Abd,

Department of Electrical Engineering,

College of Engineering, Babylon University,

Babylon, Iraq

Email: Eng.haider.jabber@uobabylon.edu.iq

\section{INTRODUCTION}

Despite there are several approaches obtainable for biomedical image segmentation, about approaches need high computational difficulty for feature extraction and segmentation [1-4] for example the technique which includes of machine learning, Artificial swarm intelligence (ASI) for example Artificial bee colony $(\mathrm{ABC})$ despite the fact approximately other techniques follow a lot of procedures for new algorithms, segmentation and classification [5-9]. The biomedical image segmentation contains of image databases, pre-processing, feature extraction, postprocessing, cataloguing, and performance estimation. The pre-processing is a development of arrangements that is implemented on image data, to complete a best presentation of a data base. It has an important influence on the act of the segmentation algorithm. Numbers 
pre-processing stage, in biomedical image processing, contains of image cropping, filtering, segmentation, gradient operations and classification [10-12]. So, it is value to state that enhancing the segmentation process will advance the performance of a designated classification method [13].

In addition, physical inspection by a prime dermatologist, skin self-investigation in at-house backgrounds is appreciated for the premature analysis of skin cancer. A full skin self-check comprises a comprehensive analysis of wholly body portions, including the scalp parts. In totaling, imaging knowledges help in accurate analysis at an initial step, chief to better behavior and organization approaches for skin cancer lesions [14]. Level still Ahn et al., [15] lectured the problematic of lesions success to the image border and completed better outcomes in evaluation to Fan et al., training [16], they did not contract with dye diagrams and dark angles in the pictures. Therefore, they purely indifferent these insufficiencies manually previously any pre-processing, which is a deadly effort. From Figure 1 display the dermoscopy images with variations in lesion features.
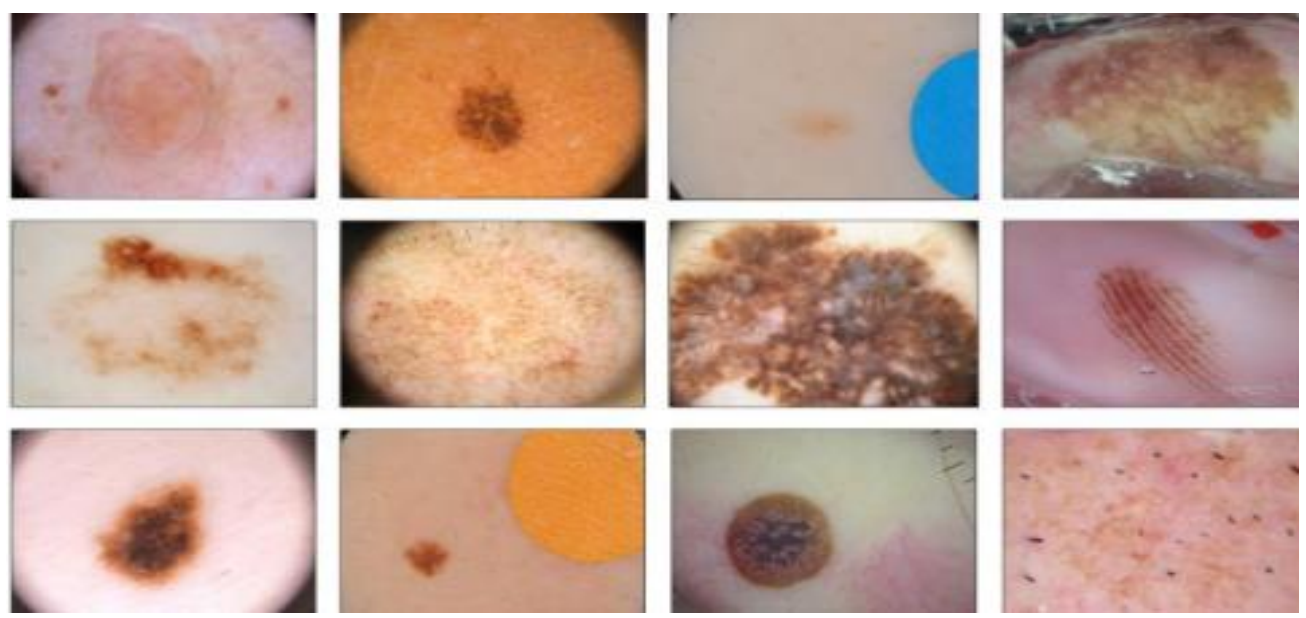

Figure 1. Dermoscopy images illustrating variations in lesion characteristics [17]

Stimulated by the foraging performance of swarm, a novel kind of optimization technique mentioned to as the artificial swarm intelligence system was technologically advanced [18]. Owing to its candidness and real affluence of operation, ASIs has been extensively used to resolve some kinds of optimization difficulties. Nevertheless, like other swarm intelligence optimization procedure, like, ABC process is good. Our area in this article is to make known to an all-purpose technique for optimal skin lesions. There are some problems in scheming such a wide-ranging algorithm. In real, dermoscopy picture databases are complex.

Maximum expected to be afforded by the kind of CPU investigation of the images designated. Images originated with many abnormalities and objects and hence it is critical to survey the proper stages and approaches defined here to medicine these irregularities and complete an exact analysis. The optimal segmentation with suitable broad-mindedness permits for satisfactory correctness in feature segmentation which in turn benefits in take full advantage of classification precision. All-purpose outline for segmentation must skilled of overcoming these problems.

\section{METHOD} construction:

The real control of $\mathrm{ABCDE}$ outline is completed for the 5-mechanisms with the subsequent

$$
\text { Sabcde }=\sum_{i=1}^{5} \text { wipi }
$$

where wi and pi are the weight and the rate competent to each estimation. By Capdehourat et al., [19], according to Stolz et al., [20]. Important that the progress of the skin cancer lesions over time is the maximum data, at that point the feature supposed by the part $\mathrm{E}$ is applied individual for graphic calculation by dermatologists at what time it is available. The assessment of ABCDE is reached resulting (1) with the points to classify the coloring into 3 -cases [19]: 


$$
\begin{array}{ll}
\text { Sabcde }<4.75 & \text { (Healthy Moles) } \\
4.75 \leq \text { Sabcde } \leq 5.75 & \text { (Suspicious Moles) } \\
\text { Sabcde }>5.75 & \text { (Unhealthy Moles) }
\end{array}
$$

In this study, we explain an automatic detection of the ASIs to variety the required investigation to segment the skin cancer of the $\mathrm{PH}^{2}$, ISIC $(2016,2017,2018)$. These approaches are a resolution-protective which leads to study high-level topographies and improve the segmentation routine. We have applied motivation function in the planned segmentation networks to classify separately pixel in the dermoscopy image into 2-parts (skin lesions and non-lesions).

The ISIC 2018 databases contains 2,594 images with the conforming GT disguises marked by professional dermatologists. The justification and challenging groups comprise 1,000 images, without ground truth. In our experimentations, we used $80 \%$ of the training traditional of the ISIC 2018 dataset for training and $20 \%$ for justification as planned in [21]. In try, ISBI 2017 databases were separated into workout, justification and challenging groups with 2000 images. Memo that we trained our typical with ISIC 2018 training groups and estimated our typical on ISBI 2016, ISBI 2017 assessment and ISIC 2018 validation groups.

We experienced our approresults on the ISIC 2018 [22] database comprising skin cancer lesions [23] including lesion images from three modules. The calculation standards are the same as those conversed in the optimal segmentation sector except that there may be some images where the target feature does not occur, and the algorithm should not be result all. Figure 2 A block diagram of the steps in the total process complicated is illustrated.

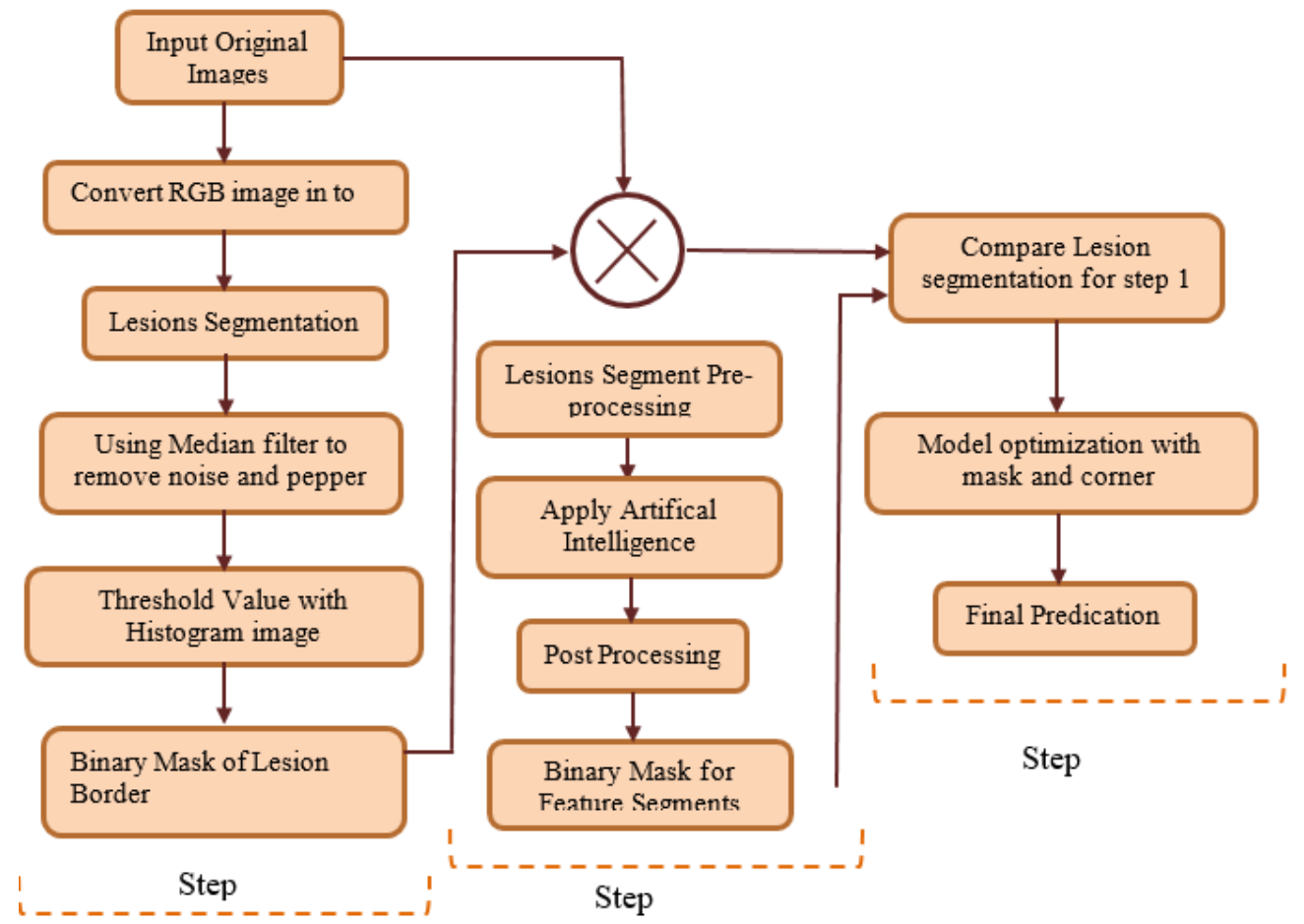

Figure 2. The flow diagram of the steps in the entire process complicated is illustrated

Estimation of the outcomes of melanoma lesions segmentation procedure can be similarly multipart and arguable in relations of recording the finest method. The boundary of skin cancer lesion segmentation differs extensively among professionals. Estimation approaches can be assessment is depending on a score on condition that by human focusses. The experimental format for counting must be correctly clear for example, the counting technique is dependable among changed subjects. An impartial assessment is depending on error quantities designed while relating the automated border with the normal manual melanoma lesion regions edge. Several objective assessment procedures are demarcated in the works [24]. 


\section{RESULTS AND DISCUSSION}

To estimate our method and the projected optimal segmentation technique, we apply four various databases. Three of these databases are designated from (ISBI) in 2016, 2017 and 2018 parts. The pictures in ISBI $(2016,2017)$ have an excessive quantity of matching matters (intelligent materials). So, we try and examination our system on each of these databases. Additional database that has been extensively applied in dermoscopic study is PH2, the codes are executed in MATLAB R2017b, and all experimentations are done on removal and any noise.

To estimate the segmentation technique quantitatively, we include DSC, jaccard similarity index (JSI), and the parameters are Accuracy (Acc), Sensitivity (Sens), and Specificity (Spec) as designated in [25]. The outcomes of challenging the projected optimal segmentation method on changed dermoscopic databases are associated to the other up-to-date methods and reported in Tables 1-4. When accomplished on the PH2, our method outclasses the other approaches in Table 1. PH2 is a comparatively minor and easy database that all its pictures were taken by a constant achievement arrangement, and it is normal to reach estimation metrics as high as informed values in Table 1. The ISBI2016 database, outcomes are described for both physical activity and challenging groups. As understood in Table 2, our algorithm outclasses the other failed detection-based algorithms $[15,16]$ and our previous technique in Zamani et al. [26]. A on the ISBI2016 train group. Its routine is a little of poorer quality than that of Yuan et al. [27], which is depending on DCNNs. Assessment of the ISBI2016 assessment group is informed in Table 2. In another way, we use our technique on the ISBI $(2017,2018)$ exam group and current the outcomes in Tables 3 and 4.

Our strategic procedure reaches average Jac value of $(96.98,94.12,88.00$ and 90.00), JSI of (94.55, $89.11,76.27$ and 79.88) on 600 images of the ISBI2017 and ISBI2018 test group, the variances from other higher competitors in Table 4 are assessment parameters have the same drift. The outcomes demonstrate that the projected technique can realize a good segmentation routine, equivalent to the other principle of approaches, which regularly are depending on DCNNs and ABC algorithm. Note that the ISBI2016, ISBI2017 and ISBI2018 physical activity groups were applied to train the failed plots given in Tables 2-4. Figure 3 illustrations the segmentation out comes and their saliency plots (the middle row). The green characterizes the GT classification, red for the curve of the lesion's primary cover, and the blue curves display the boundaries of the distinguished last segmentation through level group progression.

Table 1. Results of ph2 dataset segmentations

\begin{tabular}{llllll}
\hline Technique & Acc & Spec & Sen & Jac & JSI \\
\hline Pennisi [28] & 89.4 & 97.01 & 71.13 & - & - \\
Barata [29] & - & - & - & 91.4 & - \\
Ahn [15] & 93.67 & - & 78.76 & 89.34 & - \\
Zamani [26] & 96.58 & 98.01 & 92.94 & 92.90 & 85.88 \\
Jahanifar [30] & 97.33 & 98.55 & 97.52 & 95.22 & 92.67 \\
Proposed & $\mathbf{9 8 . 2 3}$ & $\mathbf{9 8 . 4 5}$ & $\mathbf{9 7 . 8 9}$ & $\mathbf{9 6 . 9 8}$ & $\mathbf{9 4 . 5 5}$ \\
\hline
\end{tabular}

Table 2. Results of ISBI2016 train set segmentations

\begin{tabular}{llllll}
\hline Technique & Acc & Spec & Sen & Jac & JSI \\
\hline Fan [22] & 91.40 & - & 71.13 & 81.11 & - \\
Ahn [23] & - & - & - & 83.55 & - \\
Zamani [26] & 93.51 & 98.07 & 83.2 & 89.90 & 82.08 \\
Jahanifar [30] & 94.23 & 98.45 & 88.72 & 91.72 & 85.57 \\
Proposed & $\mathbf{9 5 . 3 8}$ & $\mathbf{9 8 . 0 0}$ & $\mathbf{9 0 . 9 5}$ & $\mathbf{9 4 . 1 2}$ & $\mathbf{8 9 . 1 1}$ \\
\hline
\end{tabular}

Table 3. Results of ISBI2017 test set segmentations

\begin{tabular}{llllll}
\hline Technique & Acc & Spec & Sen & Jac & JSI \\
\hline Yuan [27] & 93.40 & 97.03 & 82.5 & 84.09 & 76.5 \\
Ahn [15] & 93.4 & 94.32 & 80.1 & 84.7 & 75.8 \\
Zamani [26] & 93.51 & 95.60 & 82.22 & 85.98 & 81.00 \\
Jahanifar [30] & 93.23 & 98.50 & 81.33 & 83.92 & 74.80 \\
Proposed & $\mathbf{9 4 . 4 0}$ & $\mathbf{9 8 . 2 0}$ & $\mathbf{9 2 . 6 2}$ & $\mathbf{8 8 . 0 0}$ & $\mathbf{7 6 . 2 7}$ \\
\hline
\end{tabular}

Table 4. Results of ISBI2018 test set segmentations

\begin{tabular}{llllll}
\hline \multicolumn{1}{c}{ Technique } & Acc & Spec & Sen & Jac & JSI \\
\hline Yuan [27] & 94.66 & 96.39 & 83.50 & 85.00 & 77.55 \\
Ahn [15] & 93.22 & 94.00 & 82.18 & 83.77 & 77.11 \\
Zamani [26] & 93.88 & 95.00 & 83.75 & 85.00 & 82.44 \\
Jahanifar [30] & 93.00 & 97.44 & 82.30 & 84.90 & 78.97 \\
Proposed & $\mathbf{9 4 . 0 0}$ & $\mathbf{9 8 . 6 5}$ & $\mathbf{9 1 . 7 6}$ & $\mathbf{9 0 . 0 0}$ & $\mathbf{7 9 . 8 8}$ \\
\hline
\end{tabular}

Int J Elec \& Comp Eng, Vol. 10, No. 6, December 2020 : 5703 - 5708 
a
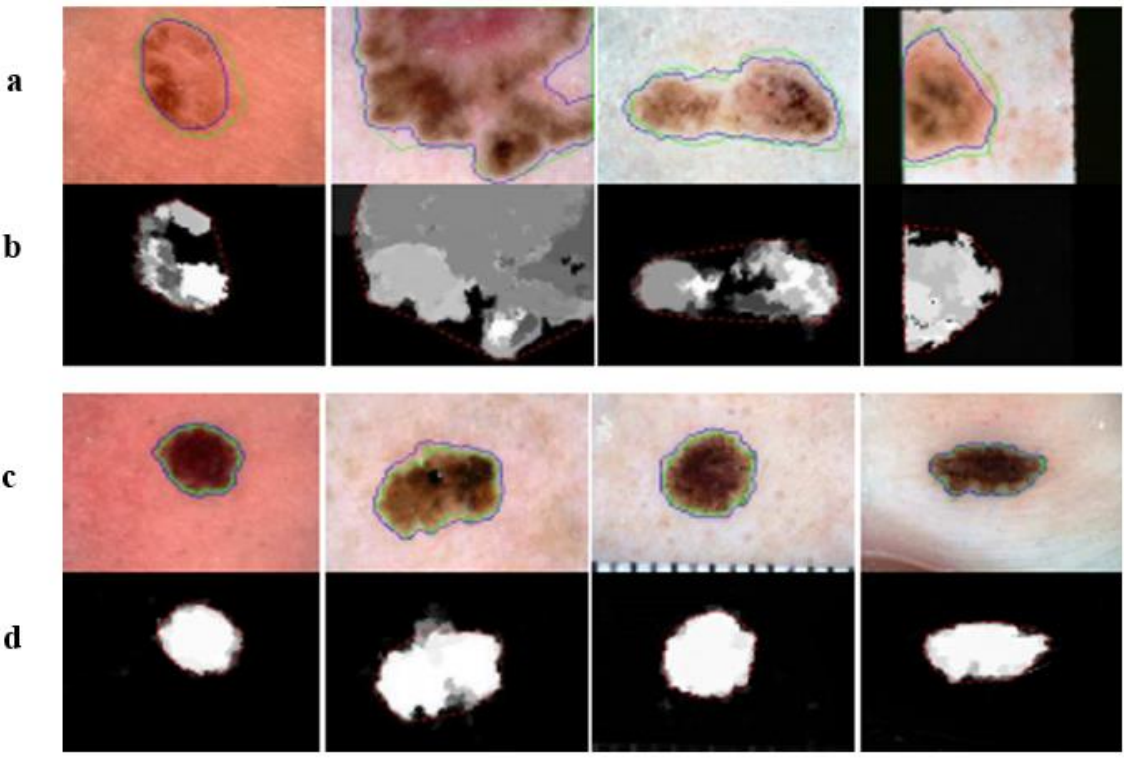

Figure 3. Sampling valuation of the proposed segmentation technique.

The ( $a$ and $b$ ) row in Figure 3 shows four examples of risky cases with their conforming the ABC technique through modern ASI with median filter. The (c and d) row in Figure 3 illustrates that data for four simple cases. In wholly pictures the red, blue, and green outlines characterize the borders of GT.

\section{CONCLUSION AND FUTURE WORK}

The methods objective at automating the checkup of skin cancer lesions, conventionally completed by dermatologists, are these days liberated by the deep-learning-built approaches.These approaches are the record precise and scalable, then they need large training databases and thus their applicability in dermatology is cooperated by the scope of the publicly dermatological databases, which are frequently slight and comprise blockings. We present a resolution for this problematic that depend on careful information decontamination that eliminates communal obstructions from dermoscopic pictures and increase that applies the modern procedure of swarm intelligence technique information generation to enhance information balancedness. They prove the efficiency of our system on the skin lesions segmentation task.

The assessment of the result is depending on the total (Acc, Sen, Spec, Jac and JSI) of the segmentation and cataloguing on the checkup group. In this field, it is very significant not to error malignance despite the fact being capable of suitably recognize benign lesions as much as probable. The goal is to mark highest Sen whereas adjusting to rise Spec, thus raising the total Acc.The work confirmations a different process for lesions optimal segmentation that could be functional to a variation of images with changed possessions and insufficiencies is proposed with multistep pre-processing stage.

\section{REFERENCES}

[1] Karaboga D, Akay B, "A comparative study of artificial bee colony algorithm," Appl Math Comput, vol. 214, pp. 108-113, 2009.

[2] Arifin AZ, et al, , "Automatic image slice marking propagation on segmentation of dental CBCT," TELKOMNIKA (Telecommunication, Computing, Electronics and Control), vol. 17, no 9, pp. 3218-3225, 2019.

[3] J. Muthuswamy, B. Kanmani, "Optimization Based Liver Contour Extraction of Abdominal CT Images," International Journal of Electrical and Computer Engineering (IJECE), vol. 8, no. 6, pp. 5061-5070, 2018.

[4] Cao Y, Lu Y, Pan X, Sun N, "An improved global best guided artificial bee colony algorithm for continuous optimization problems," Cluster Comput., vol. 22, pp. 3011-3019, 2018.

[5] M. Albkosh, et al., "Optimization of discrete wavelet transform features using artificial bee colony algorithm for texture image classification," International Journal of Electrical and Computer Engineering (IJECE), vol. 9, no. 6 , pp. 5252-5262, 2019.

[6] Karaboga D, Basturk B, "On the performance of artificial bee colony (ABC) algorithm," Appl Soft Comput, vol. 8, pp. 687-697, 2008.

[7] Mezura-Montes E, Cetina-Domínguez O, "Empirical analysis of a modified artificial bee colony for constrained numerical optimization," Appl Math Comput, vol. 218, no. 22, pp. 10943-10973, 2012. 
[8] Ma L, Zhu Y, Zhang D, Niu B, "A hybrid approach to artificial bee colony algorithm," Neural Comput Appl, vol. 27, no. 2, pp. 387-409, 2016.

[9] Kiran M. S, Hakli H, Gunduz M, Uguz H, "Artificial bee colony algorithm with variable search strategy for continuous optimization,” Inf Sci, vol. 300, pp. 140-157, 2015.

[10] Al-masni M. A, Al-antari M. A, Choi M-T, Han S-M, Kim T-S (2018) Skin lesion segmentation in dermoscopy images via deep full resolution convolutional networks," Comput Methods Prog Biomed, vol. 162, pp. 221-231, 2018.

[11] Vaishali Jaiswal, Varsha Sharma, Sunita Varma, "An implementation of novel genetic based clustering algorithm for color image segmentation," TELKOMNIKA (Telecommunication, Computing, Electronics and Control), vol. 17, no. 3, pp. 1461-1467, 2019.

[12] Kang D, Kim S, Park S, "Flow-guided hair removal for automated skin lesion identification," Multimed Tools Appl, vol. 77, no. 8, pp. 9897-9908, 2018.

[13] Sankaran S, et al., "A comparative assessment of segmentations on skin lesion through various entropy and six sigma thresholds," International Conference on ISMAC in Computational Vision and Bio-Engineering, pp. 179-188, 2018.

[14] Jayanthi Muthuswamy, B Kanmani, "Optimization Based Liver Contour Extraction of Abdominal CT Images," International Journal of Electrical and Computer Engineering (IJECE), vol 8, no 6, pp. 5061-5070, 2018.

[15] E. Ahn, J. Kim, L. Bi, A. Kumar, C. Li, M. Fulham, and D. D. Feng, "Saliency-based Lesion Segmentation via Background Detection in Dermoscopic Images," IEEE J. Biomed. Heal. informatics, vol. 21, no. 6, pp. 1685-1693, 2017.

[16] H. Fan, F. Xie, Y. Li, Z. Jiang, and J. Liu, "Automatic segmentation of dermoscopy images using saliency combined with Otsu threshold," Comput. Biol. Med., vol. 85, pp. 75-85, 2017.

[17] ISIC, "ISIC Archieve: The International Skin Imaging Collaboration: Melanoma Project," ISIC, 5 Jan 2016. [Online]. Available: https://isic-archive.com/\#.

[18] S. A. Yasear, and K. R. Ku-Mahamud, "Non-dominated sorting Harris's hawk multi-objective optimizer based on reference point approach," Indonesia Journal of Electrical Engineering and Computer Science, vol. 15, no. 3, pp. 1603-1614, 2019.

[19] Germán Capdehourat, Andrés Corez, Anabella Bazzano, Rodrigo Alonso, and Pablo Musé, "Toward a combined tool to assist dermatologists in melanoma detection from dermoscopic images of pigmented skin lesions," Pattern Recognition Letters, vol. 32, no. 16, pp. 2187-2196, 2011.

[20] W Stolz, et al, "Abcd rule of dermatoscopy-a new practical method for early recognition of malignant-melanoma," European Journal of Dermatology, vol. 4, no. 7, pp. 521-527, 1994.

[21] Salido J. A. A., "Conrado Ruiz Jr. Using deep learning to detect melanoma in dermoscopy images," International Journal of Machine Learning and Computing, vol. 8, no. 1, pp. 61-68, 2018.

[22] P. Tschandl, C. Rosendahl, and H. Kittler, "The HAM10000 dataset, a large collection of multi-sources dermatoscopic images of common pigmented skin lesions," Sci. Data, vol. 5, 2018.

[23] N. C. F. Codella, et al.. "Skin lesion analysis toward melanoma detection: A challenge at the 2017 International symposium on biomedical imaging (ISBI), hosted by the international skin imaging collaboration (ISIC)," 2018 IEEE 15th International Symposium on Biomedical Imaging (ISBI 2018), Washington, DC, pp. 168-172, 2018.

[24] Maen Takruri, Mohamed Khaled Abu Mahmoud, Adel Al-Jumaily, "PSO-SVM hybrid system for melanoma detection from histo-pathological images," International Journal of Electrical and Computer Engineering (IJECE), vol. 9, no. 4, pp. 2941-2949, 2019.

[25] N. C. F. Codella, et al., "Skin Lesion Analysis Toward Melanoma Detection: A Challenge at the 2017 International Symposium on Biomedical Imaging (ISBI), Hosted by the International Skin Imaging Collaboration (ISIC)," IEEE 15th International Symposium on Biomedical Imaging (ISBI 2018), pp. 168-172, 2018.

[26] N. Zamani Tajeddin and B. Mohammad Zadeh Asl, "A General Algorithm for Automatic Lesion Segmentation in Dermoscopy Images," 23rd Iranian Conference on Biomedical Engineering and 1st International Iranian Conference on Biomedical Engineering (ICBME), pp. 134-139, 2016.

[27] Y. Yuan, M. Chao, and Y. C. Lo, "Automatic Skin Lesion Segmentation Using Deep Fully Convolutional Networks with Jaccard Distance," IEEE Trans. Med. Imaging, vol. 36, no. 9, pp. 1876-1886, 2017.

[28] A. Pennisi, et al, "Skin lesion image segmentation using Delaunay Triangulation for melanoma detection," Comput. Med. Imaging Graph., vol. 52, pp. 89-103, 2016.

[29] C. Barata, et al, "Two Systems for the Detection of Melanomas in Dermoscopy Images Using Texture and Color Features," IEEE Syst. J., vol. 8, no. 3, pp. 965-979, 2013.

[30] M. Jahanifar, N. Zamani Tajeddin, B. Mohammadzadeh Asl and A. Gooya, "Supervised Saliency Map Driven Segmentation of Lesions in Dermoscopic Images," in IEEE Journal of Biomedical and Health Informatics, vol. 23, no. 2, pp. 509-518, Mar. 2019. 